NT@UW-04-012

\title{
Chiral solitons in nuclei: Electromagnetic form factors
}

\author{
Jason R. Smith and Gerald A. Miller \\ Department of Physics \\ University of Washington \\ Seattle, WA 98195-1560
}

\begin{abstract}
We calculate the electromagnetic form factors of a bound proton. The Chiral Quark-Soliton model provides the quark and antiquark substructure of the proton, which is embedded in nuclear matter. This procedure yields significant modifications of the form factors in the nuclear environment. The sea quarks are almost completely unaffected, and serve to mitigate the valence quark effect. In particular, the ratio of the isoscalar electric to the isovector magnetic form factor decreases by $20 \%$ at $Q^{2}=1 \mathrm{GeV}^{2}$ at nuclear density, and we do not see a strong enhancement of the magnetic moment.
\end{abstract}




\section{INTRODUCTION}

Recent polarization transfer experiments at TJNAF [1] observed a difference in the electromagnetic form factors of a proton bound in a Helium nucleus compared to a free one. This, along with other effects, such as the nuclear EMC effect [2], seems to suggest the modification of hadrons in the nuclear medium.

There is extensive work on the medium modifications of electromagnetic properties of the nucleon in the literature (for example, see Refs. [3, 4, 5, 6] ). This includes effective Lagrangians as well as models that include the quark substructure of hadrons. While in principle these effects could be couched in terms of effective field theory operators, it is our thesis that such results may be more transparent, physically intuitive or straightforward to calculate when viewed as a change in the internal structure of the hadrons.

We will use the Chiral-Quark Soliton model (CQS) 7, 8], which has a direct connection to QCD via the Instanton Liquid model, to provide our subnuclear degrees of freedom. The primary motivation is that this model includes sea quarks which we have seen to be important in the nuclear EMC effect 9]. In that case, the large medium modification in the valence quark sector is reduced through the lack of such an effect in the sea (which can be seen directly in Drell-Yan experiments [10]). The CQS is combined with the nuclear medium in a self-consistent quark-meson coupling calculation as in our previous work [9], and the electromagnetic form factors are extracted via the wave functions of the quarks using the results of Ref. 11]. The overall procedure is similar to the Quark-Meson Coupling model (QMC) [3], which uses the MIT bag model for the nucleon. The bag model does not include sea quarks. It is a confining model, whereas the CQS model is not. Additionally, the QMC model calculation, when coupled with a Relativistic Distorted Wave Impulse Approximation (RDWIA) calculation [12] or a Relativistic Multiple-Scattering Glauber Approximation (RMSGA) calculation [13, 14], improves the agreement between theory the TJNAF data 1]. With our study, we hope to reinforce the interpretation of the medium effect in terms of quark degrees of freedom, as well as provide an alternate model when the accuracy of the data is improved.

We begin with a brief description of the CQS model in Section II] In Section [II, we motivate and present our procedure to embed this model in nuclear matter. This description differs only slightly from that in our previous work [9]; it is repeated for completeness. 
Subsequently, we describe the numerical methods, and proceed to the results in Section IV.

\section{CHIRAL QUARK-SOLITON MODEL}

The CQS model Lagrangian with (anti)quark fields $\bar{\psi}, \psi$, and profile function $\Theta(r)$ is [7]

$$
\mathcal{L}=\bar{\psi}\left(i \not \supset-M e^{i \gamma_{5} \boldsymbol{n} \cdot \boldsymbol{\tau} \Theta(r)}\right) \psi
$$

where $\Theta(r \rightarrow \infty)=0$ and $\Theta(0)=-\pi$ to produce a soliton with unit winding number. The quark spectrum consists of a single bound state and a filled negative energy Dirac continuum; the vacuum is the filled negative continuum with $\Theta=0$. The wave functions in this spectrum provide the input for the electromagnetic form factors.

We work to leading order in the number of colors $\left(N_{C}=3\right)$, with $N_{f}=2$, and in the chiral limit. While the former characterizes the primary source of theoretical error, one could systematically expand in $N_{C}$ to calculate corrections. We take the constituent quark mass to be $M=420 \mathrm{MeV}$, which reproduces, for example, the $N-\Delta$ mass splitting at higher order in the $N_{C}$ expansion, as well as many electromagnetic properties [8, 11].

The theory contains divergences that must be regulated. We use a single Pauli-Villars subtraction. The Pauli-Villars mass is determined by reproducing the measured value of the pion decay constant, $f_{\pi}=93 \mathrm{MeV}$, with the relevant divergent loop integral regularized using $M_{P V} \simeq 580 \mathrm{MeV}$.

The nucleon mass is given by a sum of the energy of a single valence level $\left(E^{v}\right)$, and the regulated energy of the soliton $\left(E_{\Theta}\right.$, equal to the sum of energy levels, $E_{n}$, in the negative Dirac continuum with the sum of the energy levels in the vacuum, $E_{n}^{(0)}$, subtracted)

$$
\begin{aligned}
M_{N} & =N_{C} E^{v}+E_{\Theta}(M)-\frac{M^{2}}{M_{P V}^{2}} E_{\Theta}\left(M_{P V}\right) \\
E_{\Theta}\left(M^{\prime}\right) & =\sum_{E_{n}, E_{n}^{(0)} \leq 0} E_{n}-\left.E_{n}^{(0)}\right|_{M=M^{\prime}} .
\end{aligned}
$$

The field equation for the profile function, which follows from the Lagrangian (2.1), is

$$
\Theta(r)=\arctan \frac{\rho_{p s}^{q}(r)}{\rho_{s}^{q}(r)}
$$

where $\rho_{s}^{q}$ and $\rho_{p s}^{q}$ are the quark scalar and pseudoscalar densities, respectively, and are given by sums of the wave functions of every occupied energy level. 
The electromagnetic form factors are also given in terms of the wave functions, and are derived in Ref. [11]. The formulae are reproduced here, with a Pauli-Villars regulator, for convenience. To leading order in $N_{C}$, we have only the isoscalar electric and isovector magnetic form factors $\left(G_{X}^{T=0,1}=G_{X}^{p} \pm G_{X}^{n}\right)$

$$
\begin{aligned}
& G_{E}^{T=0}\left(q^{2}\right) \stackrel{N_{C} \rightarrow \infty}{=} \frac{N_{C}}{3} \int d \boldsymbol{r} e^{i \boldsymbol{q} \cdot \boldsymbol{r}}\left\{\sum_{E_{n} \leq E^{v}} \psi_{n}^{\dagger}(\boldsymbol{r}) \psi_{n}(\boldsymbol{r})-\sum_{E_{n}^{(0)} \leq 0} \psi_{n}^{(0) \dagger}(\boldsymbol{r}) \psi_{n}^{(0)}(\boldsymbol{r})\right\} \\
& G_{M}^{T=1}\left(q^{2}\right) \stackrel{N_{C \rightarrow \infty}}{=} \frac{N_{C} M_{N}}{3} \varepsilon^{j k l} \frac{i q^{j}}{\left|q^{2}\right|} \int d \boldsymbol{r} e^{i \boldsymbol{q} \cdot \boldsymbol{r}}\left\{\sum_{E_{n} \leq E^{v}} \psi_{n}^{\dagger}(\boldsymbol{r}) \gamma^{0} \gamma^{k} \tau^{l} \psi_{n}(\boldsymbol{r})\right. \\
& \\
&\left.-\frac{M^{2}}{M_{P V}^{2}} \sum_{E_{n}^{(P V)} \leq 0} \psi_{n}^{(P V) \dagger}(\boldsymbol{r}) \gamma^{0} \gamma^{k} \tau^{l} \psi_{n}^{(P V)}(\boldsymbol{r})\right\} .
\end{aligned}
$$

The $\psi_{n}^{(P V)}(\boldsymbol{r})$ are the solutions of the Dirac equation with the replacement $M \rightarrow M_{P V}$. In the nuclear medium, Eqs. (2.4) acquire a dependence on the Fermi momentum $G_{X}^{T=0,1}\left(q^{2}\right) \rightarrow$ $G_{X}^{T=0,1}\left(q^{2}, k_{F}\right)$ through the wave functions. This dependence is the subject of the next section.

\section{NUCLEAR PHYSICS}

We will begin with some motivation for our procedure to couple the quark substructure of the nucleon to the nuclear medium. Through the use of QCD sum rules, Ioffe [15] derived a relationship between the vacuum scalar condensate, $\langle\bar{\psi} \psi\rangle_{0}$, and the nucleon mass. One can re-derive this estimate in a constituent quark field theory such as we are using here. We begin with the scalar condensate

$$
\begin{aligned}
\langle\bar{\psi} \psi\rangle_{0} & =-\operatorname{tr} \int^{\Lambda} \frac{d^{4} p}{(2 \pi)^{4}} \frac{1}{\not p-M} \\
& \sim-\frac{N_{C} M \Lambda^{2}}{4 \pi^{2}}
\end{aligned}
$$

where the divergent integral is regulated by a momentum cutoff (playing the role of the Borel mass in the QCD sum rule approach). Using the fact that constituent quarks are essentially defined as having a mass $\sim M_{N} / N_{C}$, we can rewrite Eq. (3.1) as

$$
M_{N} \sim-\frac{4 \pi^{2}}{\Lambda^{2}}\langle\bar{\psi} \psi\rangle_{0}
$$


Although Eq. (3.2) is not a very accurate estimate, it does highlight the role of the condensate. It will be modified in the presence of other nucleons.

The condensate at finite density can be written in terms of the nuclear scalar density $\rho_{s}^{N}$ and the nucleon sigma term $\sigma_{N}[16]$ as

$$
\langle\bar{\psi} \psi\rangle_{\rho}=\langle\bar{\psi} \psi\rangle_{0}-\langle\bar{\psi} \psi\rangle_{0} \frac{\sigma_{N}}{m_{\pi}^{2} f_{\pi}^{2}} \rho_{s}^{N} .
$$

We can then substitute Eq. (3.3) into Eq. (3.2) to obtain a schematic picture of the effect of the nuclear medium on the nucleon mass

$$
M_{N}(\rho) \sim-\frac{4 \pi^{2}}{\Lambda^{2}}\left[\langle\bar{\psi} \psi\rangle_{0}-c_{s} \rho_{s}^{N}\right],
$$

where $c_{s}$ is the combination of the vacuum condensate, pion mass, decay constant and the the sigma term in Eq. (3.3).

Using this dependence of the nucleon mass on the nuclear medium as a guide, we incorporate the medium dependence in the model by simply letting the quark scalar density in the field equation (2.3) contain a (constant) contribution arising from other nucleons present in symmetric nuclear matter. This models a scalar interaction via the exchange of multiple pairs of pions between nucleons. We take the scalar density to consist of three terms: 1) the constant condensate value $\langle\bar{\psi} \psi\rangle_{0}$ (in the vacuum or at large distances from a free nucleon), 2) the valence contribution $\rho_{s}^{v}$ and 3) the contribution from the medium which takes the form of the convolution of the nucleon $\rho_{s}^{N}$ and valence quark scalar densities as in the QMC model [3]

$$
\begin{aligned}
\rho_{s}^{q}(\boldsymbol{r}) \simeq & \langle\bar{\psi} \psi\rangle_{0}+\rho_{s}^{v}(\boldsymbol{r})+\tilde{c}_{s} \int d \boldsymbol{r}^{\prime} \rho_{s}^{N}\left(\boldsymbol{r}-\boldsymbol{r}^{\prime}\right) \rho_{s}^{v}\left(\boldsymbol{r}^{\prime}\right) \\
= & \langle\bar{\psi} \psi\rangle_{0}+\rho_{s}^{v}(r)+\tilde{c}_{s} \rho_{s}^{N} S \\
& S \equiv \int d \boldsymbol{r}^{\prime} \rho_{s}^{v}\left(\boldsymbol{r}^{\prime}\right) .
\end{aligned}
$$

We take the pseudoscalar density to have only the valence term $\rho_{p s}^{q} \simeq \rho_{p s}^{v}$; the two other contributions analogous to the first and third terms of Eq. (3.5) vanish due to symmetries of the QCD vacuum and nuclear matter. These approximations to the densities neglect the precise form of the negative continuum wave functions in Eq. (2.3). The resulting free nucleon profile function has no discernible difference from a fully self-consistent treatment, demonstrating the excellence of this approximation. We take $\tilde{c}_{s}=c_{s} / S$ in Eqs. (3.4) and (3.5) to be a free parameter, which we vary to fit nuclear binding. This can be seen as either 
varying $\sigma_{N}$ in Eq. (3.3) or the vacuum value of the condensate in Eq. (3.5) with $\rho_{\Gamma}^{q} \rightarrow \rho_{\Gamma}^{q} / \tilde{c}_{s}$, as was done in Ref. 9], since the overall normalization cancels in Eq. (2.3).

The nucleon scalar density is determined by solving the nuclear self-consistency equation

$$
\rho_{s}^{N}=4 \int^{k_{F}} \frac{d^{3} k}{(2 \pi)^{3}} \frac{M_{N}\left(\rho_{s}^{N}\right)}{\sqrt{k^{2}+M_{N}\left(\rho_{s}^{N}\right)^{2}}} .
$$

The dependence of the nucleon mass, and any other properties calculable in the model, on the Fermi momentum $k_{F}$ enters through Eq. (3.6). Thus there are two coupled selfconsistency equations: one for the profile, Eq. (2.3), and one for the density, Eq. (3.6). These are iterated until the change in the nucleon mass Eq. (2.2) is as small as desired (in our case, $\Delta M_{N} \lesssim 0.1 \mathrm{MeV}$ ) for each value of the Fermi momentum. We use the KahanaRipka (KR) basis [17], with momentum cutoff $\Lambda$ and box size $L$ extrapolated to infinity (from a maximum value of $\Lambda L=150$, comparable to that in Ref. [11]), to evaluate the energy eigenvalues and wave functions used as input for the densities, nucleon mass, and electromagnetic form factors.

While the vacuum value of the condensate does not vary with the Fermi momentum by definition, the effective condensate, $\langle\bar{\psi} \psi\rangle_{0}+\tilde{c}_{s} \rho_{s}^{N}\left(k_{F}\right) S\left(k_{F}\right)$, falls $\sim 30 \%$ at nuclear density, q.v. Eq. (3.3). This is consistent with the model independent result [16] that predicts a value $25-50 \%$ below the vacuum value.

A phenomenological vector meson (mass $m_{v}=770 \mathrm{MeV}$ ) exchanged between nucleons (but not quarks in the same nucleon), is introduced as a substitute for uncalculated solitonsoliton interactions in order to obtain the necessary short distance repulsion which stabilizes the nucleus. This does not affect the form factors Eqs. (2.4a and (2.4b). The resulting energy per nucleon is

$$
\frac{E}{A}=\frac{4}{\rho_{B}\left(k_{F}\right)} \int^{k_{F}} \frac{d^{3} k}{(2 \pi)^{3}} \sqrt{k^{2}+M_{N}\left(k_{F}\right)^{2}}+\frac{1}{2} \frac{g_{v}^{2}}{m_{v}^{2}} \rho_{B}\left(k_{F}\right) .
$$

The mass of a free nucleon is computed to be $M_{N}\left(k_{F}=0\right)=1209 \mathrm{MeV}$. The $\sim 30 \%$ difference is as expected in the model at leading order in $N_{C}$. We evaluate the nucleon mass Eq. (2.2) and energy per nucleon Eq. (3.7) as a function of $k_{F}$. We choose our free parameters to fit $E / A-M_{N}(0) \equiv B=-15.75 \mathrm{MeV}$ at the minimum. We use the value $\tilde{c}_{s}=1.27$ (corresponding to $\sigma_{N}=41.4 \mathrm{MeV}$ ), and vector coupling $g_{v}^{2} / 4 \pi=10.55$, which gives a Fermi momentum of $k_{F}=1.38 \mathrm{fm}^{-1}$ in nuclear matter consistent with the known value $k_{F}=1.35 \pm 0.05 \mathrm{fm}^{-1}[18]$. We plot the binding energy per nucleon using Eq. (3.7) 
in Fig. 1. The compressibility is $K=348.5 \mathrm{MeV}$ which is above the experimental value $K=210 \pm 30 \mathrm{MeV}$, but well below the Walecka model [19] value of $560 \mathrm{MeV}$. The selfconsistent calculation results in the profile functions for zero density, $0.5 \rho_{0}, 1.0 \rho_{0}$ and $1.5 \rho_{0}$ in Fig. 2 (where $\rho_{0}$ is nuclear density).

\section{RESULTS AND DISCUSSION}

We use Eqs. (2.4a and (2.4b) to calculate the form factors, which we present in Figs. 3 and 4. We also present the results in terms of the ratios

$$
\frac{G_{E, M}^{T=0,1}\left(Q^{2}, k_{F}\right)}{G_{E, M}^{T=0,1}\left(Q^{2}, 0\right)} \equiv \frac{G_{X}^{*}\left(Q^{2}\right)}{G_{X}\left(Q^{2}\right)}
$$

where $-q^{2} \equiv Q^{2}, X$ is $E(T=0)$ or $M(T=1)$, and the double ratio

$$
\frac{G_{E}^{*}\left(Q^{2}\right) / G_{M}^{*}\left(Q^{2}\right)}{G_{E}\left(Q^{2}\right) / G_{M}\left(Q^{2}\right)} .
$$

These ratios are plotted in Figs. 5 and 6 for $0.5 \rho_{0}, 1.0 \rho_{0}$ and $1.5 \rho_{0}$.

The electric form factor is dominated by the valence contribution and shows a dramatic effect, while the magnetic form factor has equally important contributions from the valence and the sea. The latter shows almost no change in nuclear matter; it shows only a $1.3 \%$ enhancement of the magnetic moment at nuclear density, and a $2.3 \%$ enhancement at 1.5 times nuclear density. The effect in the electric form factor calculated here is comparable to that of the QMC model [3]; the main difference from that calculation lies in the lack of enhancement in the magnetic form factor, specifically the practically unchanged value of the magnetic moment.

While both form factors use the same wave functions, the isovector magnetic form factor includes an extra weighting by a factor of the angular momentum of the state (relative to the electric form factor) due to the $\gamma^{k}$ in Eq. (2.4b). This extra factor is not only responsible for making the regularization of Eq. (2.4b) necessary, but for making the sea contribution as important as the valence. In the CQS model, the orbital angular momentum carried by the sea is comparable to the orbital angular momentum carried by the valence quarks 20] (the sum of which make up about $60 \%$ of the total angular momentum of the nucleon state, with the remainder belonging to the intrinsic spin of the constituent quarks). 


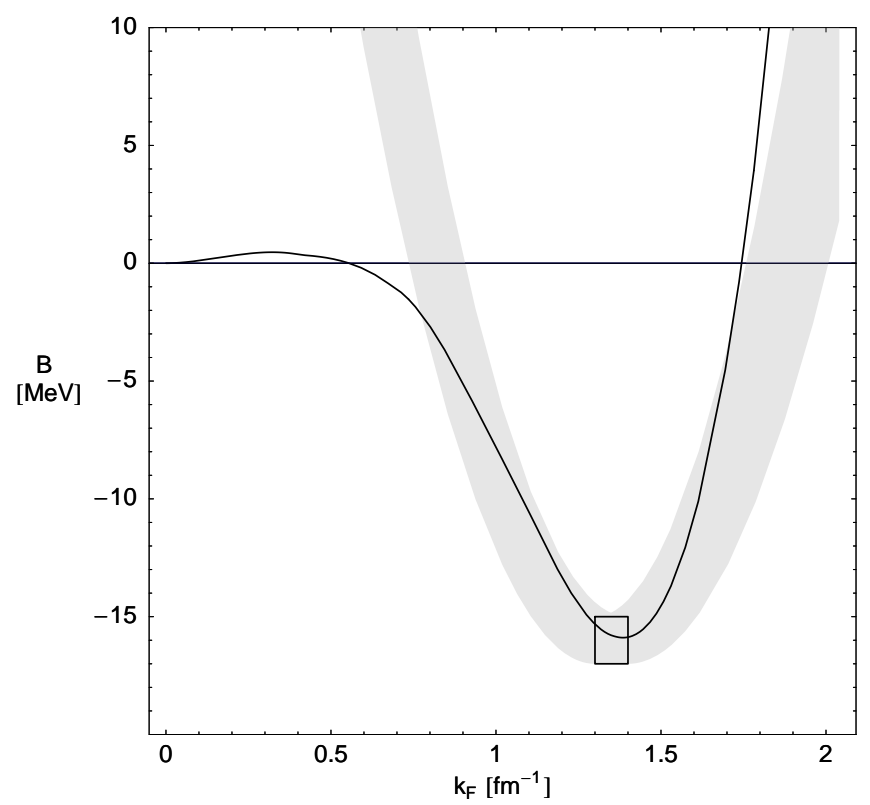

FIG. 1: Binding energy per nucleon $B=E / A-M_{N}$. The box and the gray band correspond to the uncertainty in the known values of the binding energy, density and compressibility of nuclear matter [18].

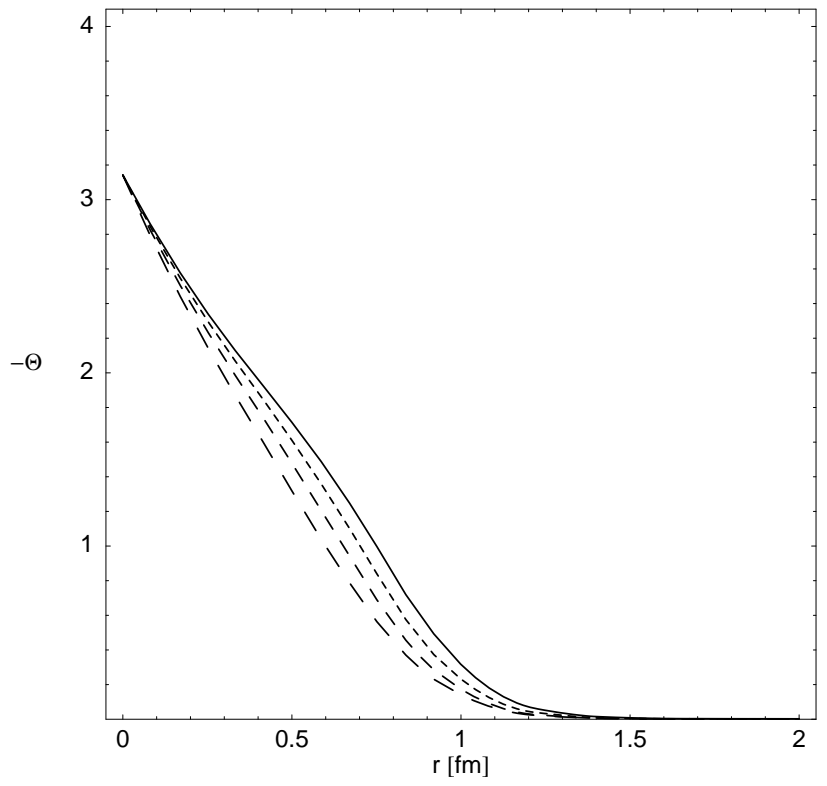

FIG. 2: Profile functions in nuclear matter. The solid line is the profile function for $1.5 \rho_{0}$; the curves with progressively longer dashes correspond to $1.0 \rho_{0}, 0.5 \rho_{0}$ and zero density respectively. 


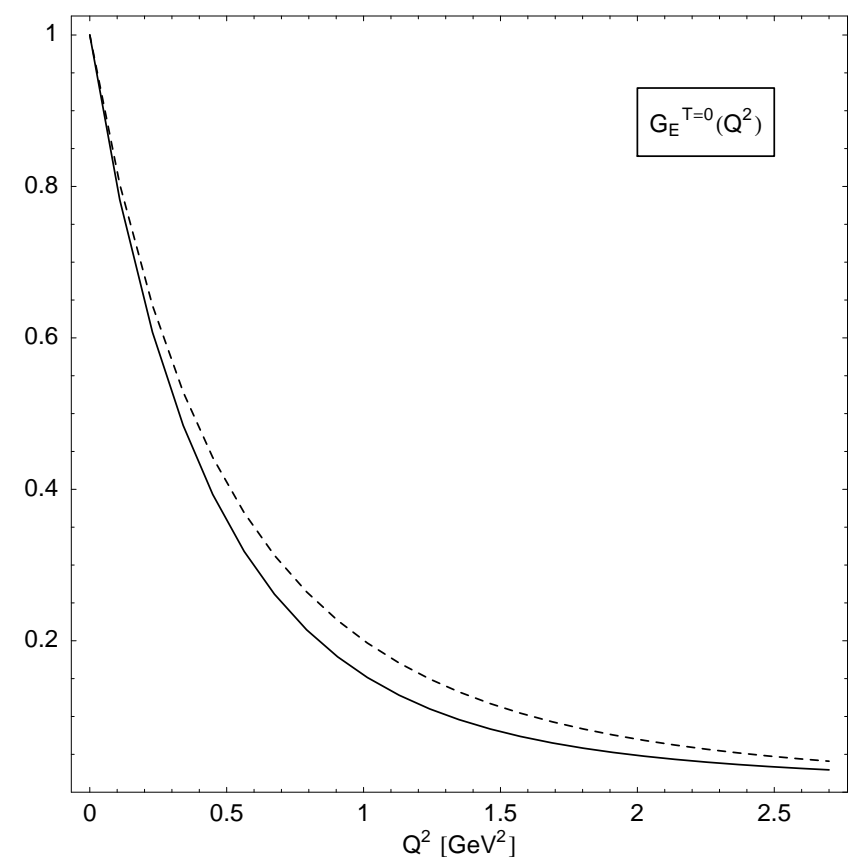

FIG. 3: The isoscalar electric form factor at nuclear density (solid) and at zero density (dashes).

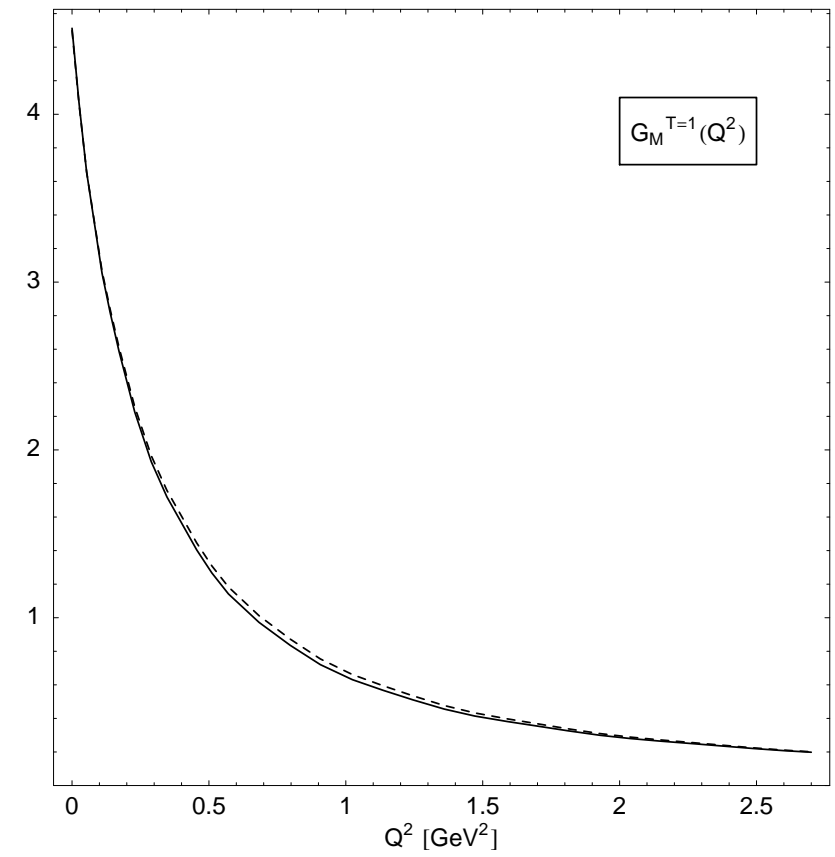

FIG. 4: The isovector magnetic form factor at nuclear density (solid) and at zero density (dashes).

Conversely, the isoscalar electric form factor (which is finite, after the vacuum subtraction) does not have as large of a contribution from the sea. The valence level is the most important piece, even at $Q^{2}>0$, since the $Q^{2}$ dependence in the form factors arises from the wave functions [11]. The negative Dirac continuum wave functions largely cancel in the 


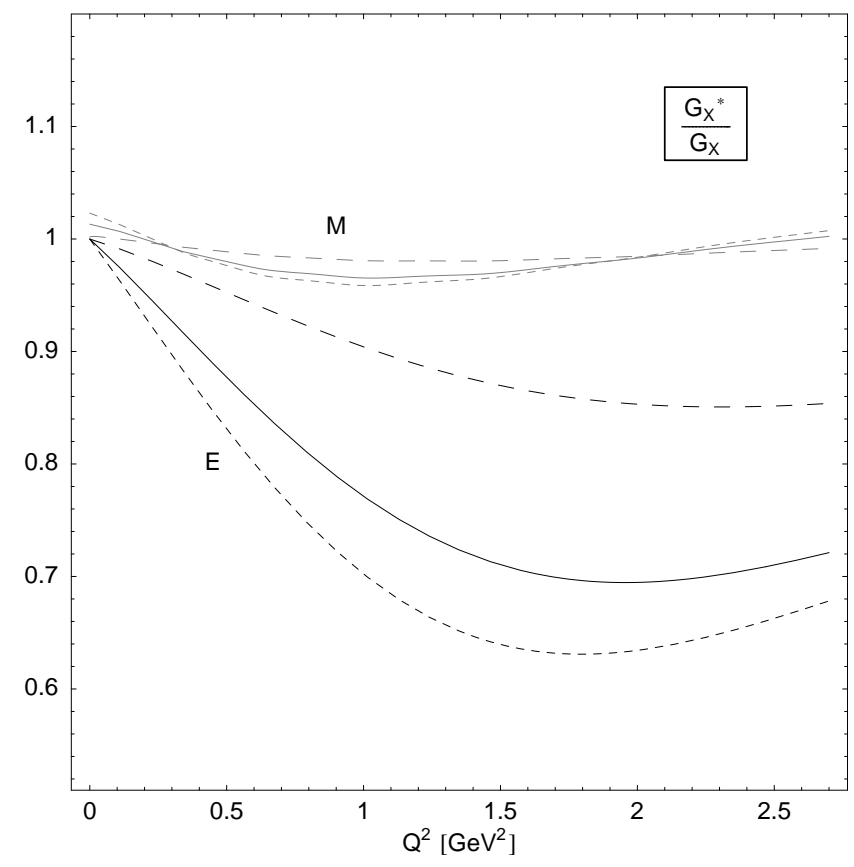

FIG. 5: The electric (lower three curves) and magnetic (upper three curves) form factor ratios in Eq. (4.1) for $0.5 \rho_{0}$ (long dashes), $1.0 \rho_{0}$ (solid) and $1.5 \rho_{0}$ (short dashes).

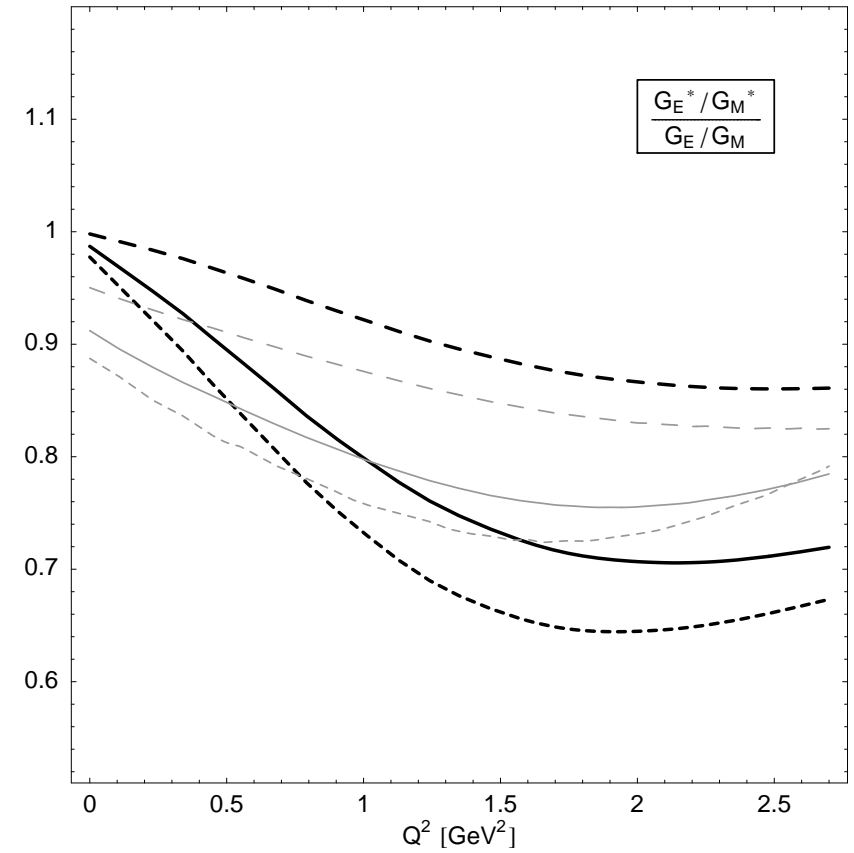

FIG. 6: The double ratio Eq. (4.2) of the electric to magnetic form factors in nuclear matter and in the vacuum from the CQS model (heavy) and the QMC model [3] (light). Three densities are shown: $0.5 \rho_{0}$ (long dashes), $1.0 \rho_{0}$ (solid) and $1.5 \rho_{0}$ (short dashes). 
vacuum subtraction in Eq. (2.4a).

The magnetic form factors are sensitive to the tail of the quark wave functions, and the mere existence of a tail is due to the lack of confinement. This is one reason for the discrepancy between the current results and the QMC model [3], but the primary source is due to the resistance to change of the sea. The former accounts for only a few percent of the difference; it is the latter that is our most important result. We see that the role of antiquarks is again prevalent as in our previous work [9].

The double ratio obtained in Fig. 6] has the same trend as the QMC model [3], but differs in the details. Since we obtain a similar double ratio, we expect to have similar results if we compare these results with the polarization transfer data 1]. This requires one to take the final state and relativistic effects into account through the use of the RDWIA [12] or the RMSGA [14], which accounts for a few percent of the discrepancy between the results for bound and free protons. A RMSGA calculation for the Helium reaction studied in Ref. [1] has been done with these CQS model results [21], and it delivers remarkably similar results to the same calculation done with the QMC model [14]. The CQS model predicts a smaller deviation than the QMC model from a Relativistic Plane Wave Impulse Approximation (RPWIA) calculation, which is taken as a baseline in Ref. 1]. While it slightly worsens the agreement with the data at $Q^{2} \lesssim 1$, the differences are of the same order of magnitude as the current experimental error, and both models under predict the observed deviation from a RPWIA calculation. At higher $Q^{2}$, the two models produce nearly identical results for Helium.

We ignore important corrections due to the rotation of the soliton that are suppressed by $1 / N_{C}$. These corrections break the $N-\Delta$ degeneracy, and improve the agreement of the vacuum form factors with experiment [11]. More relevant to the calculation presented here, these corrections do not affect the $Q^{2}$ dependence, but instead affect the normalization of the form factors [11]. However, there is no reason at that level to continue to ignore quantum fluctuations of the the pion field (quark loops), and treat the profile function as a purely self-consistent mean field. We will save this difficult problem for the future.

We have calculated the electric and magnetic form factors at leading order in $N_{C}$ at nuclear density using the CQS model. Our results help validate the apparent success of the QMC model in describing the polarization transfer experiment [1, 3], and provide a counterpoint to be distinguished when finer resolution becomes available in the data. In 
fact, the difference between the CQS model double ratio and the QMC model [3] is roughly the size as the current experimental error. Specifically, data on the bound nucleon magnetic form factor at low $Q^{2}$, particularly the magnetic moment, could serve to determine the role of sea quarks in nuclei.

\section{Acknowledgments}

We would like to thank the USDOE for partial support of this work. We would also like to thank P. Lava and J. Ryckebusch for doing the RMSGA calculation with the CQS model, and S. Strauch for acceptance averaging the results, so that a comparison with the data could be made.

[1] S. Strauch et al., Phys. Rev. Lett. 91, 052301 (2003).

[2] J. J. Aubert et al., Phys. Lett. B 123, 275 (1983).

[3] D. H. Lu, K. Tsushima, A. W. Thomas, A. G. Williams and K. Saito, Phys. Rev. C 60, 068201 (1999). D. H. Lu, A. W. Thomas, K. Tsushima, A. G. Williams and K. Saito, Phys. Lett. B 417, 217 (1998). A. W. Thomas, D. H. Lu, K. Tsushima, A. G. Williams and K. Saito, nucl-th/9807027

[4] U. T. Yakhshiev, U. G. Meissner and A. Wirzba, Eur. Phys. J. A 16, 569 (2003).

[5] M. R. Frank, B. K. Jennings and G. A. Miller, Phys. Rev. C 54, 920 (1996).

[6] E. Ruiz Arriola, C. V. Christov and K. Goeke, Phys. Lett. B 225, 22 (1989).

[7] D. Diakonov and V. Y. Petrov, in "At the Frontier of Particle Physics, Vol. 1" M. Shifman (ed.), World Scientific, Singapore, 359-415 (2001).

[8] C. V. Christov et al., Prog. Part. Nucl. Phys. 37, 91 (1996).

[9] J. R. Smith and G. A. Miller, Phys. Rev. Lett. 91, 212301 (2003) .

[10] D. M. Alde et al., Phys. Rev. Lett. 64, 2479 (1990).

[11] C. V. Christov, A. Z. Gorski, K. Goeke and P. V. Pobylitsa, Nucl. Phys. A 592, 513 (1995).

[12] J. M. Udias, J. A. Caballero, E. Moya de Guerra, J. E. Amaro and T. W. Donnelly, Phys. Rev. Lett. 83, 5451 (1999). 
[13] J. Ryckebusch, D. Debruyne, P. Lava, S. Janssen, B. Van Overmeire and T. Van Cauteren, Nucl. Phys. A 728, 226 (2003).

[14] P. Lava, J. Ryckebusch, B. Van Overmeire and S. Strauch, nucl-th/0407105.

[15] B. L. Ioffe, Nucl. Phys. B 188, 317 (1981) [Erratum-ibid. B 191, 591 (1981)].

[16] T. D. Cohen, R. J. Furnstahl and D. K. Griegel, Phys. Rev. C 45, 1881 (1992).

[17] S. Kahana and G. Ripka, Nucl. Phys. A 429, 462 (1984).

[18] J. P. Blaizot, Phys. Rept. 64, 171 (1980).

[19] J. D. Walecka, Annals Phys. 83, 491 (1974).

[20] M. Wakamatsu and H. Yoshiki, Nucl. Phys. A 524, 561 (1991).

[21] P. Lava, J. Ryckebusch, and S. Strauch, private communication. 